\title{
New antimicrobials-sequence and serendipity in San Diego
}

\author{
Jeffrey Fox
}

Luck still plays a significant role in antimicrobial drug discovery, but genomics is becoming increasingly important. This refrain was sung by a surprising number of academic and company investigators at a recent conference on antimicrobial agents and chemotherapy*. However microbial genomics is but one segment of a rapidly expanding trove of new approaches for identifying new microbial drug targets and rapidly making and testing compounds for activity against them.

The new molecular approaches to studying pathogens not only help "to identify targets, but also create more opportunities for serendipity," says C. Kendall Stover of PathoGenesis (Seattle, WA). Accordingly, Stover, his colleagues at PathoGenesis, and their collaborators at the University of Washington (Seattle, WA) are determining the genomic sequence of Pseudomonas aeruginosa, a pathogen that infects the lungs of cystic fibrosis patients as well as burn patients and wounds. The use of genomic information to provide insights into the molecular mechanisms of drug resistance will be particularly important, Stover believes.

Along these lines, Microcide Pharmaceuticals (Mountain View, CA) is discovering new antibiotics by revisiting and improving "known entities" among familiar antibiotics. The search for improved or new antimicrobial products is "iterative," involving an evaluation of which microbial genes are essential for a pathogen to survive in its animal host and then testing compounds that target those essential microbial components. Reasonable targets of a pathogen include metabolic function and stress response genes as well as specific virulence factors, says Microcide's Jerry Buysse .

Researchers at Novalon Pharmaceuticals (Durham, NC) are using peptide ligands as part of a "bio-key" assay for probing potential antimicrobial targets, says the company's Dana Fowlkes. "Peptides are used purely as probes for discovery while we screen large libraries of conventional compounds." Novalon investigators now screen up to 30 thousand compounds daily, looking for high-affinity inhibition of specific microbial enzymes.

Investigators at 3-D Pharmaceuticals (Exton, PA) are also screening vast numbers of potential inhibitors-in their case, using temperature-sensitive fluorescent reporter molecules to detect slight differences in target protein unfolding patterns when those proteins bind ligands. Roger Bones of 3-D says his colleagues may look at 50 million compounds built from about 20 distinct chemical skeletons. Making no bones, he contends this approach is "ideal for genomic targets because it does not require knowledge of the target protein's function.'

University-based researchers are also pursuing essential genes, some of which may represent new drug targets in microbial pathogens. These researchers are also developing several strategies for studying bacterial pathogens that may lead to new antimicrobial products and improved vaccines.

John Mekalanos and his colleagues at Harvard Medical School (Boston, MA) have developed a transposon-based footprinting

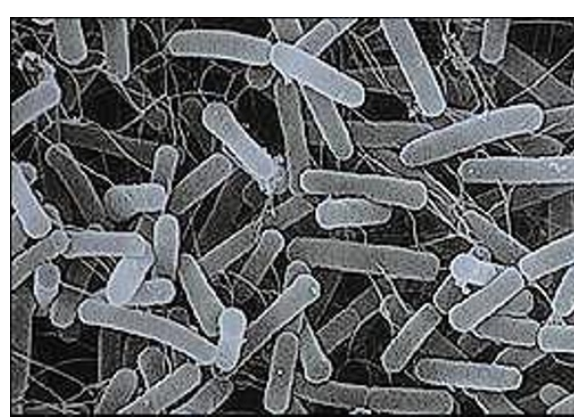

Figure 1. With antibiotic-resistant bacteria on the rise, novel methods are urgently needed to develop new classes of antimicrobials.

technique to map essential genes systematically. "We are now moving around the entire Haemophilus influenzae chromosome to look for essential genes," he says. The method enables them to detect which of those genes are needed for growth in a host animal, examining DNA segments as large as 20 kilobases. "Within the next few years, we'll have all the genes that are essential for pathogens to grow in vitro, but it will take longer to analyze those needed in vivo and to develop drug targets," he says. Mekalanos' group is following a similar approach to studying Streptococcus pneumoniae.

Jacqueline Shea, working in David Hogan's laboratory at the Hammersmith Hospital (London, UK), is using an approach called signature tag mutagenesis to identify mutant pathogens that fail to survive in animal hosts. Although the strat- egy is different from that of Mekalanos and his colleagues, the aim is similar-to identify those genes required by a particular pathogen to overcome a host's defenses. It relies on the use of "unique DNA tags of variable 40 base-pair sequences and consistent flanking sequences," she says. Thus far this approach has helped to identify new sets of virulence genes, including genes that permit the pathogen Salmonella typhimuri$u m$ to continue replicating in mammalian macrophage cells.

Also interested in obtaining such insights, Stanley Falkow and his colleagues at Stanford University (Palo Alto, CA), have developed a way to introduce altered versions of the green fluorescence protein (GFP) into pathogens in order to track their behavior in infected host tissues and cells and identify the genes involved. GFP can be used as a signal for rapidly sorting and analyzing thousands of infected mammalian cells automatically. "We are using the technique to isolate genes that are turned on only after infection," he says. "We can detect a single microbial cell in an infected animal, and can even ask what subtype of host cell is infected."

Elsewhere at Stanford, Gary Schoolnik and Michael Wilson have designed a comprehensive set of probes to study gene expression in Mycobacterium tuberculosis, the pathogen responsible for tuberculosis. From this general approach of examining differential arrays of mRNA molecules, they identified several clusters of genes involved in fatty acid synthesis that appear to confer resistance to specific antibiotics, according to Wilson. In addition, they are looking at those genes that are missing or not functioning in BCG, an attenuated strain of this bacterium that is used widely as a vaccine. "You don't need genomic sequences to do this, but it sure makes it a lot easier," he says.

Little doubt remains about the need to develop new classes of antibacterial drugs to combat the increasing number of resistant infections. With the success of a genomic approach to design neuraminidase inhibitors against flu viruses, expectations are growing that these avenues will soon begin to pay off for antimicrobials.

\footnotetext{
*The 38th Interscience Conference on Antimicrobial Agents and Chemotherapy (ICAAC), San Diego, CA, September 24-27, 1998.
} 